\title{
El proceso de una investigación cualitativa longitudinal sobre la transición a la maternidady paternidad en España
}

The process of a longitudinal qualitative research on the
transition to motherhood and fatherhood in Spain

Carmen Botía-Morillas

Universidad Pablo de Olavide (UPO) cbotmor@upo.es (ESPAÑA)

Teresa Jurado-Guerrero

Universidad Nacional de Educación a Distancia (UNED)

tjurado@poli.uned.es (ESPAÑA)

Recibido: 16.03 .2018

Aceptado: 22.08 .2018

\section{RESUMEN}

Se expone el proceso metodológico de una investigación cualitativa longitudinal y colaborativa sobre la transición a la maternidad y paternidad en España. Se ha entrevistado a parejas bi-activas en dos momentos de su ciclo vital, durante el embarazo de su primer bebé, y entre año y medio y dos años posteriores al nacimiento. Esta investigación longitudinal aporta cuatro importantes valores añadidos: 1. Estudiar los cambios de ideas, planes y actitudes sin los sesgos de memoria selectiva que suele producir el acceso a información retrospectiva; 2. Trabajar en equipo y de forma colaborativa permite obtener una amplia muestra formada por dos submuestras homogéneas socioeconómicamente, pudiendo acceder a diversidad de discursos con enorme potencialidad analítica; 3. Estudiar a ambos miembros de las parejas -sus prácticas, percepciones y justificacionesenriquece el análisis frente a estudios que cuentan con una sola perspectiva, y permite un análisis crítico de temas sujetos a sesgos de corrección política, como los ligados al género, maternidad y paternidad; 4. Evidenciar, explicitar y justificar el proceso metodológico avala la calidad y validez de la investigación sustantiva, aportando información de archivo y trastienda. 


\title{
PALABRAS CLAVE
}

Método cualitativo, entrevista, relaciones de pareja, sociología del género, validez investigación cualitativa.

\begin{abstract}
We present the methodological process of a qualitative, longitudinal and collaborative research on the transition to motherhood and fatherhood in Spain. We have interviewed double income couples in two time of their life cycle, during pregnancy of the first baby and between one year and a half and two years after birth. This longitudinal research has four advantages 1 . The changes of ideas, plans and attitudes can be studied without biases of selective memory, something usual in the access to retrospective information. 2. Working as a team and in a collaborative way allows us to obtain a wide sample formed by two socioeconomically and homogeneous sub-samples, being able to access the diversity of discourses with enormous analytical potential. 3. Studying both members of the couples, -their practices, perceptions and justifications-, enriches the analysis compared to studies that have only the perspective of one partner, and allows us to better analyze topics that suffer from biases of political correctness such as those related to gender, motherhood and fatherhood. 4.Demonstrating, specifying, and justifying the methodological process guarantees the quality and validity of the substantive research, providing information of archive and back room.
\end{abstract}

\section{KEY WORDS}

Qualitative method, interview, couple relationships, sociology of gender, qualitative research validity.

\section{INTRODUCCIÓN}

El artículo presenta el proceso metodológico de una investigación cuyo objetivo ha sido analizar la transición a la primera maternidad y primera paternidad durante los años 2011 y 2013 en España. La investigación forma parte de un estudio internacional más amplio que trata de analizar y comprender por países, un objetivo de investigación común: cuáles son los cambios sociales y relacionales que suceden en las parejas cuando llega su primer bebé, así como comprender el significado de estos cambios para cada uno de los miembros de la pareja. En España se ha seguido una aproximación metodológica cualitativa y longitudinal en la que se ha entrevistado a 58 parejas de doble ingreso, heterosexuales y heterogéneas socio-económicamente, con ambos miembros económicamente activos. 
Se les ha entrevistado en dos momentos de su ciclo vital: durante el embarazo, momento en el que esperaban la llegada de su primer bebé y cuando el bebé tenía entre 18 y 24 meses de vida.

El artículo muestra los procesos metodológicos llevados a cabo y la toma de decisiones adoptadas, trata de ofrecer evidencias que respaldan la estrategia metodológica seguida, para presentarla no sólo al ámbito académico, sino a la ciudadanía como principal beneficiaria de los hallazgos de una investigación social con pretensión de responsabilidad social, transparencia, disponibilidad y accesibilidad a todo el proceso investigador. Así se ha intentado, ya que el diseño metodológico, la dirección de los procesos de investigación y el análisis de resultados no agotan el trabajo a realizar desde la sociología, sino que tanto práctica como teóricamente el proyecto de investigación, su planteamiento y la reflexión teórica sobre su objeto -la definición de la demanda, en palabras de Zárraga (2009: 40)-, son fundamentales para que la investigación no sea una acumulación de datos empíricos sin significado (Zárraga 2009). Este artículo trata de responder a la importancia de vincular el proceso metodológico seguido, su explicitación y justificación, a los conceptos de calidad y validez de la investigación cualitativa, explicando qué se pretendía, cómo se ha procedido y justificando por qué.

El análisis presentado en el artículo se divide en ocho apartados: 1. el método seguido, 2. la selección muestral, 3. el trabajo de campo realizado, 4. el proceso de contactación, 5. la entrevista repetida, 6. la fidelización de las parejas, 7.el proceso analítico y 8 . las conclusiones.

\section{UNA INVESTIGACIÓN CUALITATIVA LONGITUDINAL: OBJETIVO Y JUSTIFICACIÓN}

\subsection{El objeto de análisis como demandante de la estrategia metodológica}

Como objeto de investigación, se han tratado de comprender y profundizar en los procesos sociales implicados en el momento del ciclo vital analizado. Como Ortí (2001) afirma, no es posible elegir entre los tres posibles cometidos de la sociología: comprender el mundo, transformarlo o simplemente administrarlo, ya que en toda investigación están articuladas estas tres dimensiones. Así, para poder comprender es imprescindible una aproximación cualitativa al objeto de investigación, ya que la investigación cualitativa permite entender los fenómenos sociales desde la perspectiva de los actores, examinar el modo como experimentan el mundo y conocer los aspectos importantes de la realidad para quien la percibe (Taylor y Bogdan 2002). Centrarse en el análisis de la realidad cotidiana y de las prácticas de cada varón y mujer justo en el momento de su transición hacia su primera maternidad y paternidad ha sido importante para explicar las pequeñas y grandes decisiones de la vida y las formas prácticas como la resuelven en lo cotidiano: sus estrategias, interacciones, conflictos, motivaciones, los roles desempeñados, sus ambigüedades, prioridades, la importancia del 
empleo, de la familia, aspectos todos ellos que precisan una mirada cualitativa y comprensiva. Esto no sucede en el vacío, sino que son parejas inmersas en estructuras sociales, permitiendo el enfoque cualitativo centrarse en la causalidad estructural (Martín Criado 2014a), lo que aporta un importante valor añadido al proceso metodológico que lo hace posible. En la investigación no se ha seguido una perspectiva idealista que reduce el lenguaje a expresión sino una perspectiva pragmática del lenguaje, como plantea Martín Criado (1998), a partir de Goffman (1963; 1974) y Bourdieu (1985). Así, los sujetos sociales producen discursos condicionados por sus esquemas cognitivos, adquiridos en su trayectoria social y en las situaciones sociales en las que interactúan. Esta ha sido la perspectiva metodológica seguida en la investigación.

\subsection{Por qué no la estrategia cuantitativa}

No se optó por seguir la estrategia cuantitativa, precisamente por sus implicaciones ontológicas, como adoptar un realismo ingenuo que asume que la realidad que interesa investigar es la que se puede medir. Cuando se realizan estudios estadísticos con encuestas representativas se asumen ciertas lógicas de acción individual y de interacción entre las personas. Como ejemplo, en los estudios de familia hay teorías que asumen la unión de intereses en las parejas, mientras que otras la conciben como un lugar de negociación de intereses e ideas individuales. Muchos estudios cuantitativos siguen usando los mismos supuestos acerca de las estrategias familiares de hace medio siglo, como unión de intereses, a pesar de que en las últimas décadas la sociedad española ha vivido profundas transformaciones sociales y familiares, como el control de fecundidad y el acceso de las mujeres a la educación y al empleo, lo que va ligado a nuevas estrategias personales y familiares.

Además, hay tres cuestiones presentes en las encuestas con cuestionario relevantes para nuestro objeto de investigación, por las que no se ha considerado apropiado seguir esta técnica cuantitativa. La primera de ellas, es el sesgo de deseabilidad social, muy presente en las encuestas; la segunda es que las encuestas permiten un nivel de detalle mucho menor, al solicitar a las personas encuestadas que elijan entre una serie de opciones de respuesta ya previamente establecidas, y de algún modo restringidas; y en tercer lugar, las encuestas no permiten captar los procesos, quizá sólo el antes y el después en estudios longitudinales; pero que haya una relación entre dos aspectos no implica necesariamente que podamos conocer los procesos por los que los aspectos que se relacionan llegan a asociarse con el tiempo. Para profundizar en otros presupuestos de la encuesta, véase, Merllié (1993).

Veamos un ejemplo concreto sobre la dificultad de estudiar el tema del conflicto en las tareas domésticas con encuestas. Un estudio estadístico encontró una relación significativa entre la frecuencia de discusiones sobre las tareas domésticas y su reparto. Cuanto más se discutía menos se implicaba el hombre en las tareas (Jurado-Guerrero, González y Naldini 2012). Este resultado era con- 
trario a la hipótesis de trabajo que hacía esperar que una negociación explícita del reparto de las tareas lograra un reparto más equilibrado. El reparto de tareas domésticas es un proceso cotidiano, en el que una parte puede demandar más colaboración a la otra y ésta reaccionar positivamente (conflicto transformador) o ésta hacer caso omiso o reaccionar colaborando solo puntualmente (conflicto recurrente sin transformación). El estudio cualitativo de este tipo de procesos permite comprender resultados aparentemente contradictorios derivados de las encuestas, como el del anterior ejemplo.

\subsection{La investigación cualitativa longitudinal (ICL) con una misma muestra}

La investigación cualitativa en España ha seguido diversidad de paradigmas, perspectivas estratégicas y diseños de investigación determinados por la demanda de cada una de las investigaciones realizadas. La investigación cualitativa inicial estaba muy influida por la personalidad y forma de hacer de los sociólogos cualitativos que la desarrollaron, en muchos casos centrados en la técnica cualitativa del grupo de discusión (véase Ibáñez 1979; Lucas 1992; Ortí 2014). Alonso y Rodríguez (2014) consideran al cualitativismo crítico español, precursor de la investigación cualitativa en España, como una respuesta a los límites de la investigación social empírica del momento, una respuesta abierta y crítica con el orden social y con su transformación, desde una amplia imaginación sociológica, para un contexto social y político muy concreto. No obstante, quizá se ha mitificado la posibilidad de transformación y radicalidad de la sociología cualitativa (Ortí 2007). Debe diferenciarse la Investigación Cualitativa Longitudinal (ICL) con una misma muestra, la desarrollada en el presente texto, de al menos las siguientes:

a. la investigación cualitativa sincrónica para un objetivo de investigación transversal y para una población específica en un momento temporal concreto; es la investigación más frecuente.

b. la investigación cualitativa diacrónica o longitudinal con una muestra distinta para cada año de estudio, representada en España por la Investigación Cualitativa Continua (ICC) de Zárraga (2009), en la que entre 1979 y 1982 se analizaron 268 grupos de discusión para analizar opiniones y actitudes de la población sobre la situación política del momento y su evolución.

La estrategia metodológica adoptada es la investigación cualitativa longitudinal (ICL) o diacrónica con una misma muestra. Esta estrategia está aumentando en los últimos años para objetos de investigación similares (véase Fox 2009 y Wiesmann, Boeije, Doorne-Huiskes y Dulk 2008, o Botía-Morillas 2011, para España; sin olvidar estudios clásicos más antiguos como Hochschild y Machung 1989). También está aumentando la ICL en general, véase Caïs, Folguera y Formoso 2014, o Colectivo IOE 2011, sobre inserción de alumnado inmigrante iberoamericano en el sistema educativo español. 
Nuestra unidad de análisis es la pareja, observando a varones y mujeres por separado, otros estudios cualitativos españoles también tienen a la pareja como unidad de análisis a partir de entrevistas en profundidad. Entre estos, el de Gutiérrez (2002), que estudia la negociación en la pareja e identifica cuatro tipos de parejas en función de cómo relacionan los ámbitos público, privado y doméstico. Díaz, Díaz, Dema e Ibáñez (2004) que estudian cómo el empleo de varón y de la mujer influyen en la dinámica interna de la familia. Dema $(2005 ; 2006)$ que analiza la negociación en torno al dinero y afirma que la independencia económica de las mujeres no garantiza la igualdad en la pareja. Y en un estudio más reciente (Prieto 2015) analiza el uso de los tiempos desde la perspectiva de las mujeres. Otros estudios españoles, sobre un objeto de investigación similar, y diferente unidad de análisis y de estrategia metodológica son, entre otros, los numerosos estudios sobre el reparto de las tareas domésticas y cuidados, pero la mayoría se abordan desde una perspectiva cuantitativa. Algunos combinan esta estrategia junto a la cualitativa, como Tobío $(2001 ; 2005)$ que analiza la percepción de las madres trabajadoras sobre sí mismas y la dificultad de enfrentarse a las responsabilidades familiares y de empleo; o Alberdi (2003), que aborda la importancia que las mujeres otorgan al empleo, a partir de grupos focales con mujeres profesionales de éxito.

Desde nuestro punto de vista, la estrategia metodológica que proponemos incorpora como novedoso para el contexto español, el abordar las relaciones de género con respecto al trabajo remunerado, no remunerado y a los cuidados en su articulación con las relaciones madre-criatura, padre-criatura y la relación entre la pareja, mediante un estudio de panel, longitudinal y cualitativo, con una misma muestra. Se trata de un estudio en el que se han entrevistado a los futuros padres durante el embarazo y cuando el bebé tenía entre un año y medio y dos años, manteniendo la misma muestra en ambos periodos. Esto permite estudiar la dimensión temporal y la dimensión transversal del proceso de interacción entre el sujeto y la estructura. En este caso se ha analizado la construcción de género en hombres y mujeres que viven en pareja y son económicamente activos en relación con la diversidad de las condiciones de vida marcadas sobre todo por el origen social, por las trayectorias formativo-laborales y en relación con un cambio biográfico decisivo para las relaciones de género: el nacimiento de su primera criatura. Entrevistar a un mismo sujeto en dos momentos de su ciclo vital permite captar la transformación de su discurso y de sus prácticas, debido al impacto del nacimiento. Esta estrategia tiene varias ventajas sustantivas y metodológicas. Primero, permite comparar los ideales iniciales con las prácticas posteriores, especialmente las justificaciones de las incoherencias entre ambos momentos. Asimismo quien entrevista y el sujeto entrevistado pueden distanciarse de sus primeras interpretaciones y emociones, pudiendo atenuarse así posibles efectos adversos de subjetividad. Construir la información a través de un panel longitudinal en vez de con preguntas retrospectivas minimiza las distorsiones del recuerdo selectivo que suele producir la construcción de información del pasado a partir del presente. También permite estudiar mejor las preferencias adaptativas que reducen las disonancias cognitivas (Merton 1948; Leahy y Doughney 
2006). Por último, una segunda entrevista permite aprovechar la confianza que se ganó la primera vez y poder reforzarla durante el segundo encuentro.

\section{LA MUESTRA, POTENCIALIDADES Y LIMITACIONES}

La muestra se diseñó siguiendo el principal objetivo de conocimiento del estudio: conocer los cambios sociales y relacionales que suceden en las parejas cuando llega su primer bebé, así como comprender el significado de estos cambios para cada uno de los miembros de la pareja. Para ello partimos de un marco teórico previo, aunque también se trataba de formular nuevas ideas teóricas. Los dos criterios básicos de la selección muestral han sido: primero, estar esperando el primer hijo, sin que ninguno de los miembros de la pareja hubiese tenido hijos anteriormente $\mathrm{y}$, segundo, que ambos estuviesen empleados o, si hubiesen perdido el empleo, que se encontrasen buscándolo activamente, este matiz ha sido necesario debido a las dimensiones del desempleo en España durante la crisis económica que comenzó en el año 2008. La llegada del primer hijo representa un reto para el reparto del trabajo remunerado y no remunerado cuando ambos miembros de la pareja son económicamente activos.

A partir de los dos criterios de selección básicos: actividad de ambos miembros de la pareja y estar esperando el primer bebé, se trató de diversificar la muestra, como recomienda Bertaux (1993) para lograr resultados consistentes para un mismo contexto de investigación. Los criterios adicionales de diversificación muestral fueron: diversidad de niveles educativos, respecto a la disponibilidad temporal y territorial.

Así, en primer lugar, se buscaron mujeres de diferentes niveles educativos; específicamente mujeres con título universitario o grado superior de formación profesional, así como mujeres por debajo de ese nivel. En España, estos dos grupos de mujeres tienden a mostrar patrones de empleo diferentes, ya que las primeras suelen alcanzar altas tasas de empleo sostenidas en el tiempo, mientras que las segundas suelen alcanzar tasas de empleo menores y tienden a sufrir más transiciones de entrada y salida del mercado de trabajo en sus edades centrales (Garrido 2004).

El segundo de los criterios adicionales de diversificación muestral se ha centrado en la disponibilidad de tiempo del hombre en la primera entrevista -y específicamente la posibilidad de volver a casa antes de las 18 horas o, al menos, a una hora temprana varios días a la semana. Los hombres que trabajan muchas horas y tienen poco tiempo libre tenderán a involucrarse menos en las tareas domésticas y en el cuidado cuando llegue la criatura, a no ser que posteriormente cambien sus condiciones de trabajo, algo improbable.

Sobre el tercer criterio de diversificación, la territorialidad, ciertamente todos fueron contextos urbanos, diversificando entre ciudades de gran y medio tamaño -Madrid, Barcelona, Sevilla y Pamplona, ya que el tamaño de la ciudad podría influir en las posibilidades de conciliar familia y empleo. La mayor dificultad de lograr conciliar familia, empleo y vida personal motivó la no elección de parejas 
provenientes de entornos rurales, en los que hay mayor proximidad entre los tres ámbitos vitales.

La estratificación de la muestra, siguiendo estos tres últimos criterios adicionales de diversificación, ha tenido como consecuencia un número de entrevistas más alto de lo que suele ser la práctica en investigaciones cualitativas. Esto no significa que la muestra sea representativa de la población española en su conjunto, ya que las entrevistadas son parejas heterosexuales de doble ingreso, que no representan la diversidad de modelos parentales presentes actualmente en la sociedad española, pero pesamos que sí se trata de una muestra suficientemente amplia con pretensión de heterogeneidad, dentro de la homogeneidad básica de partida. De hecho podría considerarse formada por dos submuestras: una más amplia de parejas de clase media, con algunas parejas representativas de la clase media-alta, con nivel educativo superior y empleos cualificados, y bastante estables, específicamente para las mujeres. Y una segunda submuestra más reducida y compuesta por parejas representativas de la clase media-baja, clase baja o clase trabajadora, con empleos menos estables, pero centrales también para varones y mujeres. Son empleos relevantes en tanto que son generadores de un salario necesario, más que por ser empleos enriquecedores o que ofrezcan buenas condiciones laborales.

El Apéndice 1 muestra a todas las parejas según el nivel educativo de él y ella, la ocupación de él y ella, los recursos relativos medidos como ingresos netos mensuales y el intervalo de ingresos de ambos como proxy del origen social al que pertenecen; información fundamental para conocer la posición de cada pareja en la estructura social, algo fundamental para poder realizar un análisis cualitativo de las prácticas e interacciones de cada pareja a partir de su contexto social.

\section{LA CONTACTACIÓN}

La Investigación Cualitativa Longitudinal (ICL) realizada se distingue además, por su carácter de investigación colaborativa mediante el trabajo en un equipo de nueve sociólogas y sociólogos, y una psicóloga social. Todas las investigadoras se han responsabilizado de todas y cada una de las fases del proceso de investigación, asumiendo una responsabilidad especifica en la contactación y realización de entrevistas por ciudades. La decisión de no externalizar el trabajo de campo permitió controlar todo el proceso de investigación desde el diseño de la misma, por parte de todo el equipo: la contactación, el seguimiento y fidelización de las parejas, las entrevistas de prueba, las entrevistas definitivas, y el análisis de los discursos. Este trabajo colaborativo permitió incorporar las cuestiones mejorables con el desarrollo de la investigación además de facilitar un conocimiento más profundo de los sujetos entrevistados. El trabajo colaborativo requirió continuas reuniones de trabajo conjuntas, tanto presenciales como a distancia. 
Las parejas fueron contactadas mediante diferentes estrategias, siguiendo las recomendaciones de acceso al campo de Hammersley y Atkinson (1994), intentando que este proceso fuese lo menos directivo posible. La principal estrategia de contactación fue a través de clases de preparación al parto, recordemos que en España estas clases son generalizadas y ofrecidas por la sanidad pública mayoritariamente; así como algunas clases de gimnasia o yoga para embarazadas. Para la principal estrategia de contactación se procedió del siguiente modo: una vez iniciado el contacto con un centro de salud o centro de educación maternal, se presentaba el proyecto de investigación a los especialistas, matronas o personal médico, y se concertaba una fecha para presentar el proyecto a las parejas en cursos de preparación al parto o sesiones informativas para padres y madres primerizas. Tras ello, se presentaba el proyecto en una breve exposición (unos 5-10 minutos) -sin mencionar nada relativo a la igualdad de género o de oportunidades, como se explica más adelante- y se repartía un tríptico con información general sobre el objetivo del proyecto: los cambios en las parejas cuando llega el primer bebé. Tras la presentación se administraba un formulario para que las parejas que estaban dispuestas a participar anotasen su número de teléfono y dirección de correo electrónico. En otros casos, la pareja, gracias al tríptico repartido, contactaba posteriormente con el investigador o investigadora de referencia en su ciudad. Más tarde, quien presentó el proyecto se encargaba de contactar con la pareja para acordar una fecha de entrevista o, en su defecto, descartarle si finalmente decidían no participar. La mayoría de las personas contactadas en un primer momento fueron mujeres, ya que ellas eran también mayoría en casi todos los cursos. Se hizo el máximo esfuerzo para motivar a la participación a parejas de diferente origen social. Por esa razón se contactó con matronas y especialistas médicos en distritos que representaban diferentes realidades socioeconómicas, y se realizaron sesiones informativas en centros públicos y privados. A pesar de los esfuerzos mencionados, estos no permitieron representar todo el espectro social, sobre todo en sus extremos (mujeres con nivel educativo bajo y mujeres de altos ingresos). Precisamente por eso, se siguieron dos estrategias más de contactación para diversificar la muestra: recurso a redes sociales (para 13 parejas) para ampliar la diversidad social de las parejas en las cuatro ciudades de estudio ${ }^{1}$, y además, dos parejas ya contactadas sirvieron de enlace con otras dos parejas, siguiendo así la estrategia bola de nieve. Estas estrategias de contactación secundarias permitieron contar con parejas de ingresos muy altos y de ingresos bajos, no obstante las parejas de clase media fueron las más presentes en la muestra.

Todas han sido parejas alejadas del modelo más tradicional de varón proveedor y madre cuidadora, ya que las mujeres son proveedoras también, ya sean principales, iguales o secundarias, además de ser, en mayor o menor medida, también cuidadoras cuando llega la maternidad. En el caso de los hombres, son proveedores todos ellos, aunque algunos se encuentren desempleados, pero este

${ }^{1}$ En ninguno de los casos contactados a través de redes sociales, la pareja entrevistada tenía relación previa con quien les entrevistó. 
no es su único rol, ya que muchos de los varones se convirtieron en padres cuidadores cuando llegó su bebé.

Para que una pareja entrase en la muestra era imprescindible que ambos miembros estuviesen de acuerdo en participar, ya que si alguno era reticente, podía peligrar el trabajo de campo, al ser difícil que ofreciera un discurso sincero, y ser probable que abandonasen la investigación para la segunda ola. La razón más frecuente que ofrecían las parejas para declinar la entrevista era la reticencia del varón a participar en el proyecto o la dificultad de encajar la entrevista con los horarios de él. Las entrevistas se celebraron en los hogares de las parejas ${ }^{2}$, contando así con información de su contexto de vida, lo que ayudó en el análisis. Sólo en el caso de tres parejas se concertaron las citas en un bar tranquilo o una biblioteca, ya que las parejas se sentían más cómodas en un espacio público.

\section{LA TÉCNICA: LA ENTREVISTA REPETIDA}

La técnica de investigación utilizada ha sido la entrevista con guion temático. En cada entrevista se han tratado los mismos temas, aunque la amplitud haya podido variar, ya que cada entrevista constituye un intercambio comunicativo único y no reproducible. La entrevista con guion temático ha permitido la comparabilidad entre los miembros de la pareja, entre las distintas parejas y la comparación territorial. Ha interesado acceder al conocimiento sobre las prácticas que se desarrollaban en el seno de cada pareja y el cambio que se va produciendo en las prácticas y en el modo de verlas, asumirlas y explicarlas. Priorizar el conocimiento sobre las prácticas, en lugar de las opiniones sobre las prácticas permite acceder al conocimiento sobre las trayectorias vitales en conjunto, y desde sus prácticas conocer las relaciones socioestructurales de las que forman parte, siguiendo así las recomendaciones de Bertaux (1993) y Bertaux-Wiame (1993) en su investigación sobre las historias de vida del oficio de panadero en Francia.

Debido al interés sobre las prácticas discursivas, descartamos el grupo de discusión; este es apropiado cuando el objeto principal de investigación son las construcciones simbólicas en torno a las prácticas, pero nos dice poco sobre las prácticas cotidianas de cada participante -sólo se cuenta en el grupo aquello que es apropiado para defender el argumento que se sostiene en la conversación(Martín Criado 1997; 2004). Si hubiéramos optado por la observación participante, tendríamos que haber estado presentes en la vida cotidiana de cada pareja antes del nacimiento y durante el primer año de vida, al menos durante diversos

${ }^{2}$ A todas las parejas se les gratificó con un detalle por su buena predisposición a ofrecernos los testimonios de sus vidas. En la primera ola se les regaló un libro sobre embarazo y cómo enfrentarse a los cuidados de un valor de 30 euros: MURKOFF, H., EISENBERG, A., y S. HATHAWAY, S. (2010): Qué se puede esperar del primer año, Barcelona, Ediciones Medici. ISBN: 978-84-9799-043-1. A las tres parejas con pocos recursos, contactadas por redes sociales para aumentar la diversificación muestral se les gratificó con un cheque regalo por valor de 50 euros. En la segunda ola se agradeció la participación de todas las parejas con una botella de aceite de oliva virgen gourmet de 500 mililitros. 
períodos, lo cual habría supuesto un trabajo de campo tan intensivo que ciertamente era inviable en la práctica de un proyecto de investigación colaborativo en un territorio tan amplio. Tampoco eran apropiados los grupos triangulares, ya que no se trataba de analizar el proceso de construcción discursiva en la interacción que se genera en un espacio social (Conde 2008).

Las entrevistas se llevaron a cabo en dos etapas y en dos momentos diferentes. En primer lugar, se realizaron entrevistas piloto entre noviembre y diciembre de 2010 para evaluar los diferentes modelos de entrevistas (el modelo basado en dos entrevistas individuales con cada uno de los miembros de la pareja versus el modelo basado en dos entrevistas individuales y una entrevista conjunta con la pareja) para poner a prueba la bondad de los temas incluidos en el guion. Antes de la segunda ola se probó también el guion con varias parejas en noviembre de 2012. Las entrevistas definitivas se realizaron durante los años 2011 a 2013 en dos momentos del ciclo vital de las parejas: durante el embarazo y cuando el bebé tenía entre 18 y 24 meses de vida. Las entrevistas de la primera ola supusieron 136 entrevistas individuales, a cada mujer y cada varón por separado, y 68 entrevistas realizadas a ambos conjuntamente. Primero se entrevistaba a uno de ellos, después al otro por separado y se concluía con la entrevista conjunta, las tres en la misma sesión. Durante la segunda ola se consideró prioritario contar con el punto de vista de cada uno de los miembros de la pareja por separado, y se entrevistó a cada miembro de la pareja individualmente.

Las entrevistas individuales permitieron conocer la biografía personal e indagar en las interpretaciones subjetivas, mientras que la entrevista conjunta permitió observar la interacción de ambos cónyuges, además de proporcionar información relativa a la historia y prácticas conyugales. Específicamente, pueden resumirse los temas sobre los que versaban los guiones de la primera y segunda ola en los siguientes:

- En las entrevistas de la primera ola se trató con cada persona por separado su trayectoria educativa y laboral, sus condiciones de trabajo, sus expectativas para su futuro laboral y sus rutinas diarias. Se habló también acerca del deseo y la decisión de tener un hijo, cómo imaginaban la vida cuando el bebé hubiese llegado, su ideal acerca de cómo cuidar de la criatura o de cómo lo haría su cónyuge y su ideal con respecto a la dedicación a su empleo. En la entrevista conjunta se trató la historia residencial y de formación de la pareja, su división del trabajo doméstico, la deseabilidad y disponibilidad de ayuda doméstica y sus planes respecto al cuidado, en particular el uso de las licencias parentales, de servicios de educación infantil y el apoyo que esperaban recibir de las y los abuelos o de otros familiares cercanos.

- En las entrevistas de la segunda ola se trató lo acontecido desde la entrevista anterior: el parto, los primeros meses, la vuelta al trabajo, la relación entre maternidad y paternidad con el empleo, la importancia del trabajo remunerado, el reparto de las tareas domésticas, los recursos para el cuidado, el tiempo con el bebé y la satisfacción con la vida cotidiana y con el tiempo disponible para estar con la pareja, los deseos de cambio y 
los planes de futuro. Asimismo se habló con ellos sobre cómo valoraban

las políticas existentes y cómo les gustaría que cambiasen.

Tras las entrevistas, se le administró un cuestionario sociodemográfico final, para las dos fases de la investigación, sobre información básica como fecha de nacimiento, semana de embarazo o fecha de parto, nivel educativo, condiciones laborales, y especialmente el nivel de ingresos y gastos mensuales de la vivienda, para así asegurar una mínima información básica común para todas las parejas, y poder conocer su perfil socioeconómico individual y de pareja. Esta información normalmente ya estaba presente en las entrevistas, pero procedimos así como práctica de investigación de control muestral y calidad global de la investigación ${ }^{3}$.

Antes de comenzar las entrevistas se agradeció a cada miembro de la pareja su participación; se les pidió permiso para grabar y se les recordó la duración aproximada de cada entrevista, una hora y media se les decía, así como la dinámica que se seguiría, tomando como modelo el de una conversación. Después de cada entrevista, se elaboró una ficha "post-entrevista", en la que se valoraba la dinámica y calidad de la misma, el desarrollo de cada tema tratado, sus dificultades y bondades, además de redactar notas sobre el contexto de vida de la pareja y el desarrollo del trabajo de campo realizado.

\section{FIDELIZACIÓN DE LAS PAREJAS}

La fidelización de las parejas entre la primera y segunda fase del trabajo de campo se ha considerado fundamental para velar por la participación de todas las parejas de la muestra en la segunda ola. Esta comenzaba ya con la información y el trato dado a cada pareja desde el primer contacto, cuando se les informaba del objetivo general del proyecto -los cambios en las parejas cuando llega el primer bebé-, sin especificar mucho más, ya que podría haber condicionado su discurso. Se informaba que se realizaría una entrevista durante el embarazo y otra cuando el bebé tuviese más de un año, por lo que ya conocían y estaban de acuerdo en participar en la segunda fase. Además, cada investigadora o investigador llamó y escribió a cada una de las parejas que había entrevistado para interesarse por el nacimiento y felicitarles, sólo en pocos casos no pudo contactarse por teléfono en este momento, felicitándoles solamente por escrito. La felicitación por escrito incluía un pequeño libro para bebés. Se considera este seguimiento como una práctica de investigación fundamental para garantizar la fidelización de las parejas, ya que ha permitido que las 68 parejas se sientan importantes, lo que era

${ }^{3}$ Puede accederse a mayor información de archivo y trastienda de la investigación realizada como el tríptico informativo utilizado en la captación de las parejas, los guiones temáticos utilizados en la primera y segunda ola de entrevistas; el cuestionario sociodemográfico administrado a cada miembro de la pareja tras concluir su entrevista; o el libro de códigos utilizados en la codificación de la $1^{\mathrm{a}}$ y $2^{\mathrm{a}}$ ola, entre otros documentos, a través del siguiente enlace: http://transparent. upf.edu/".. 
cierto, así les recordábamos que les acompañábamos en el momento vital tan relevante que estaban viviendo.

En un estudio de panel, longitudinal, como este, existe el riesgo de perder una parte de las parejas a lo largo del estudio, aunque se realice un buen seguimiento de cada pareja. En esta ICL ha habido diez parejas que no se han podido entrevistar después del nacimiento, porque se habían mudado de ciudad (una), porque se habían separado (una), porque el número de teléfono ya no estaba activo (dos) o porque los maridos no accedieron directa o indirectamente una segunda entrevista (seis). De las diez parejas a las que no se pudo hacer una segunda entrevista, siete son de nivel educativo bajo, en cinco de estas parejas ella es la que tiene un nivel educativo básico, y en dos de ellas, también él lo tiene, lo que muestra de nuevo las dificultades para acceder a los estratos de población de clase media-baja o baja. Siete de estas diez parejas se habían auto-ubicado en la primera ola como una pareja con reparto de tareas tradicional (él hace menos de un $40 \%$ ); en seis de estas siete parejas, los maridos prefirieron no continuar, podría ser plausible que se negaran a participar precisamente porque se sintiesen alejados del ideal igualitario y se sintiesen cuestionados en su realidad cotidiana y en sus puntos de vista, tras hacerse una idea de los objetivos del proyecto durante las entrevistas de la primera fase, no obstante, no podemos conocer realmente las razones.

\section{CÓMO SE HAN ANALIZADO LAS ENTREVISTAS}

Antes de evidenciar cómo se ha procedido es imprescindible recordar el objeto de investigación, y es que la clave de un buen análisis es lo que hayamos sido capaces de introducir en el diseño de investigación, debiendo la investigación sociológica cualitativa acceder a la estructura discursiva subyacente presente en las relaciones sociales (Zárraga 2009: 41). El análisis realizado fue flexible, procediendo analíticamente en tres fases, en primer lugar se realizó un análisis de contenido para disipar angustias, debido a la enorme cantidad de información y a los diez investigadores que componían el equipo de investigación. Posteriormente hubo un segundo nivel de análisis estructural, identificando y comprendiendo las estructuras subyacentes de las prácticas contenidas en los discursos. En tercer lugar se realizó un análisis sociohermenéutico o interpretativo contextualizado; centrado en la vida, dinámica e interacciones cotidianas, donde centra Alonso (1998) el análisis sociológico del discurso. Este análisis, para Ortí (2014) es un: "análisis contextual donde los argumentos toman sentido en relación con los actores que los enuncian" (p. 27).

El análisis ha partido de los comentarios post-entrevistas, compartiendo así en equipo los comentarios escritos por quien realizó la entrevista a cada pareja. En segundo lugar se leyeron e interpretaron las trascripciones literales de las entrevistas, repartiéndolas entre el equipo; y finalmente para esta primera aproximación, se tuvo en cuenta la información proporcionada por los cuestionarios socio-demográficos. En esta primera fase de análisis, se codificaron las 
entrevistas transcritas, hubo reuniones de trabajo para decidir y elaborar entre todo el equipo el libro de códigos que sería utilizado, siendo esté algo distinto para la primera y segunda ola de entrevistas. Se utilizó el programa de análisis cualitativo Atlas.ti 6.0 en su forma de uso colaborativo, debido a la cantidad de información disponible. El libro de códigos, tanto descriptivos como analíticos, se probó en una codificación interpares, para cada una de las fases, para valorar así su bondad y adecuación a los discursos de las entrevistas. Tras las pruebas interpares de codificación se redefinieron algunos códigos, y finalmente se consensuó el libro de códigos definitivo, tanto para la primera ola como para la segunda. Tras la codificación de las entrevistas de la primera y segunda ola cada miembro del equipo investigador elaboró un documento con comentarios post-codificación, una síntesis con los contenidos y cuestiones que juzgaba más relevantes para el análisis de las entrevistas de cada pareja. En estos documentos post-codificación se incluyó un resumen del contexto social y económico, los principales temas tratados, las características específicas de cada pareja e información procedente del cuestionario sociodemográfico. Este documento fue importante para realizar los análisis posteriores, ya que permitió comprender el significado de la información contenida en cada código dentro de su contexto. En una segunda fase, el análisis de las entrevistas no se basó meramente en una interpretación de algunos códigos de manera agrupada, sino que los discursos se analizaron en su conjunto para cada persona y cada pareja según el contexto global de cada pareja, para lo que fue necesario leer las entrevistas en su totalidad y relacionar sus prácticas con la estructura que las hace posibles.

La tercera fase del análisis se realizó tras las dos primeras aproximaciones analíticas anteriores, en subequipos de análisis de tres o cuatro investigadores. Cada subequipo ha desarrollado análisis específicos en función de cuatro dimensiones en las que interesaba indagar - maternidad, paternidad, reparto de tareas domésticas rutinarias y conciliación-. Los análisis han triangulado la información proveniente de las entrevistas de ellas, de ellos, de ambas olas, y de ambos de la primera ola. El trabajo en equipo y subequipos permitió manejar más rápidamente la ingente cantidad de material empírico recabado. En esta tercera fase de análisis final por subequipos, se comenzaron leyendo de nuevo las entrevistas completas y debatiendo en grupo las ideas que parecían más relevantes para los objetivos analíticos de cada subequipo. Tras este debate se escribían las primeras interpretaciones que después se confrontaban de nuevo para todas las parejas que englobase el análisis ${ }^{4}$. Se analizaron los discursos de los sujetos entrevistados de forma iterativa -yendo de los datos a la teoría y de la teoría a los datos- hasta llegar a una interpretación satisfactoria para cada subequipo de análisis (Timmermans y Tavory 2012). Estos análisis por subequipos, posteriormente eran confrontados en el equipo global.

${ }^{4}$ Todos los análisis realizados en esta tercera fase en subequipos partieron de todas las parejas de la muestra, excepto el análisis sobre las tareas domésticas, en el que se analizaron solamente las parejas que no tenían un reparto de tareas tradicional -33 de las 68 parejas iniciales. 
En línea con las propuestas metodológicas de Martín Criado (2014b) se ha partido de una teoría de la acción que concibe al sujeto como actor que piensa y actúa según sus ideas, pero constreñido por el contexto, la situación y la socialización tanto primaria como secundaria, es decir, que se adapta discursivamente a sus prácticas. Por ello, para el análisis de los discursos se tuvieron en cuenta las restricciones que pesan sobre las personas entrevistadas, especialmente las ideas y actitudes del otro miembro de la pareja, las condiciones laborales propias y de su pareja y el contexto institucional (permisos, servicios, derechos laborales).

En los análisis longitudinales de la tercera fase de análisis, se analizaron además tanto las coherencias como las incoherencias entre lo dicho durante el embarazo y lo hecho después. Asimismo se describieron los posicionamientos frente a referentes significativos como los expertos en salud materno-filial, la familia, los compañeros de trabajo y los amigos, cuando estos nos ayudaban a entender las normas sociales dominantes y el esfuerzo de algunas parejas para cuestionarlas y de-construirlas. También se intentó prestar atención a lo no dicho y a lo dado por hecho. La amplitud muestral ha sido de gran ayuda para ello, pues se han observado similares discursos y sus correspondientes prácticas unas veces acompañadas de justificaciones y otras veces como hechos dados e incuestionados. Para conocer algunos de los resultados del análisis véase Abril et al. (2015a) y González y Jurado-Guerrero (2015b).

Así, en síntesis, el proceso de análisis ha sido diverso, por etapas y realizado por todo el equipo de forma colaborativa. Primero se comenzó por un proceso analítico más intuitivo, a partir de los comentarios post-entrevista, leyendo las entrevistas, posteriormente seguimos un proceso más estandarizado: elaboración del libro de códigos, pruebas de codificación interpares, codificación de las entrevistas y elaboración de comentarios post-codificación. En tercer lugar, se realizó un análisis sociológico de los discursos en subequipos de trabajo. Es decir, las cuestiones micro, de cada pareja y de cada sujeto, se han tratado primero de forma exploratoria, yendo en un segundo momento a los contextos que ha generado cada uno de los discursos, volviendo a leer e interpretar de nuevo las entrevistas como un todo, ya alejándonos de los códigos específicos de partes del discurso. Se sigue así la perspectiva de Bertaux (1993:163) cuando afirma que el análisis no es la última fase de la investigación, se va construyendo progresivamente gracias a imaginación sociológica y no tanto a procedimientos técnicos.

\section{CONCLUSIONES: CALIDAD Y VALIDEZ DE LA INVESTIGACIÓN}

Nuestra intención ha sido desgranar la toma de decisiones adoptadas sobre todas las partes del proceso metodológico llevado a cabo, pensamos que hacerlo así, puede generar un importante valor añadido, ya que permite evidenciar, explicitar y justificar estas decisiones metodológicas, constituyéndose como 
una buena práctica al servicio de la comunidad académica y de la ciudadanía, y un criterio de mejora de la validez de la investigación cualitativa longitudinal llevada a cabo.

En la Tabla 1 se sistematizan los elementos evidenciados y justificados a lo largo del presente artículo, que, desde nuestro punto de vista avalan la calidad y validez del proceso de investigación realizado. Algunos son específicos de la investigación cualitativa longitudinal, y otros están vinculados a la validez de la investigación cualitativa en general; todos ellos componen y avalan la calidad global de la investigación presentada.

\begin{tabular}{|c|c|c|}
\hline $\begin{array}{l}\text { Características ICL rea- } \\
\text { lizada }\end{array}$ & Valor añadido & $\begin{array}{l}\text { Inconvenientes o limita- } \\
\text { ciones }\end{array}$ \\
\hline $\begin{array}{l}\text { Equipo amplio ( } 10 \text { personas) } \\
\text { que ha participado en el pro- } \\
\text { ceso responsabilizándose del } \\
\text { mismo desde el inicio hasta } \\
\text { el final. }\end{array}$ & $\begin{array}{l}\text { Diferentes perspectivas teóricas } \\
\text { y de conocimiento, reflexión } \\
\text { conjunta, cambios y mejoras so- } \\
\text { bre la marcha y posibilidad de } \\
\text { obtener heterogeneidad, dentro } \\
\text { de la homogeneidad muestral. }\end{array}$ & $\begin{array}{l}\text { Esfuerzo de coordinación, guion } \\
\text { temático común, compromisos } \\
\text { para asegurar comparabilidad } \\
\text { intra y transnacional. Mayor } \\
\text { necesidad de tiempo, de personal } \\
\text { investigador y de presupuesto. }\end{array}$ \\
\hline $\begin{array}{l}\text { Proceso de contactación por } \\
\text { diversas vías: a través de } \\
\text { profesionales de la salud y de } \\
\text { redes sociales }\end{array}$ & $\begin{array}{l}\text { Acceso a parejas fuera del } \\
\text { ámbito social del equipo de } \\
\text { investigación. Esmerado trato } \\
\text { permite fidelizar a las parejas. }\end{array}$ & $\begin{array}{l}\text { Encontrar parejas excepcionales, } \\
\text { como parejas muy igualitarias, } \\
\text { de la élite o de estratos sociales } \\
\text { desfavorecidos. }\end{array}$ \\
\hline $\begin{array}{l}\text { Muestra heterogénea y diversa } \\
\text { de parejas heterosexuales, } \\
\text { esperando el primer bebé y } \\
\text { bi-activas }\end{array}$ & $\begin{array}{l}\text { Dos submuestras homogéneas y } \\
\text { mayor diversidad en la muestra } \\
\text { global, algo novedoso para la } \\
\text { investigación cualitativa. }\end{array}$ & $\begin{array}{l}\text { Mayor esfuerzo personal por } \\
\text { ampliar el trabajo de campo y el } \\
\text { trabajo de análisis para establecer } \\
\text { resultados y conclusiones con- } \\
\text { sistentes. }\end{array}$ \\
\hline Gratificación y seguimiento & $\begin{array}{l}\text { Fidelización e incentivo para } \\
\text { participar, recuerdo para } 2^{\mathrm{a}} \text { ola }\end{array}$ & $\begin{array}{l}\text { Coste económico, influencia } \\
\text { normativa. }\end{array}$ \\
\hline $\begin{array}{l}\text { Entrevistas individuales ( } 136 \\
\text { en } 1^{\mathrm{a}} \text { ola, } 116 \text { en } 2^{\mathrm{a}} \text { ola) }\end{array}$ & $\begin{array}{l}\text { Intimidad y relativa libertad de } \\
\text { expresión. }\end{array}$ & $\begin{array}{l}\text { Los sujetos construyen sus prác- } \\
\text { ticas discursivas en interacción } \\
\text { con otros. }\end{array}$ \\
\hline $\begin{array}{l}\text { Entrevistas conjuntas ( } 681^{a} \\
\text { ola) }\end{array}$ & $\begin{array}{l}\text { Visibilización de dinámicas de } \\
\text { pareja. }\end{array}$ & $\begin{array}{l}\text { Posible censura estructural por } \\
\text { parte de la pareja. }\end{array}$ \\
\hline $\begin{array}{l}\text { Entrevistas a ambos miembros } \\
\text { de la pareja por separado }\end{array}$ & $\begin{array}{l}\text { Confrontación de discursos de } \\
\text { ella con los de él. }\end{array}$ & $\begin{array}{l}\text { Mayor esfuerzo económico y de } \\
\text { tiempo. }\end{array}$ \\
\hline Características ICL realizada & Valor añadido & Inconvenientes o limitaciones \\
\hline
\end{tabular}




\begin{tabular}{|c|c|c|}
\hline Comentarios post-entrevista & $\begin{array}{l}\text { Contextualización de la pareja, } \\
\text { necesaria para analizar su posi- } \\
\text { ción estructural. }\end{array}$ & $\begin{array}{l}\text { Puede predisponer el posterior } \\
\text { análisis. }\end{array}$ \\
\hline $\begin{array}{l}\text { Cuestionario socio-económico } \\
(68+58)\end{array}$ & $\begin{array}{l}\text { Datos sistemáticos sobre } \\
\text { recursos y perfiles sociodemo- } \\
\text { gráficos. }\end{array}$ & $\begin{array}{l}\text { Información sensible que se ad- } \\
\text { ministra al final de entrevista. }\end{array}$ \\
\hline Transcripciones literales & $\begin{array}{l}\text { Análisis del conjunto de la } \\
\text { narración. }\end{array}$ & Costoso en tiempo y dinero. \\
\hline $\begin{array}{l}\text { Estudio cualitativo de panel o } \\
\text { longitudinal (2011 y 2013) }\end{array}$ & $\begin{array}{l}\text { Menor sesgo de memoria, } \\
\text { observación de preferencias } \\
\text { adaptativas y de disonancias } \\
\text { cognitivas. }\end{array}$ & $\begin{array}{l}\text { Riesgo de mortalidad de panel (de } \\
68 \text { se han perdido } 10 \text {, sin pérdida } \\
\text { de heterogeneidad social). }\end{array}$ \\
\hline Seguimiento de parejas & Mayor confianza con el tiempo. & Costoso en tiempo y esfuerzo. \\
\hline $\begin{array}{l}\text { Codificación de entrevistas } \\
\text { con Atlas.ti }\end{array}$ & $\begin{array}{l}\text { Ayuda para almacenamiento, } \\
\text { búsquedas sistemáticas y com- } \\
\text { probación de hipótesis, y evitar } \\
\text { angustias. }\end{array}$ & Costoso en tiempo y esfuerzo. \\
\hline Comentario post-codificación & $\begin{array}{l}\text { Generación de primeras ideas } \\
\text { analíticas, contextualización. }\end{array}$ & Costoso en tiempo y esfuerzo. \\
\hline $\begin{array}{l}\text { Análisis sociológico de discur- } \\
\text { so en equipo y subequipos }\end{array}$ & $\begin{array}{l}\text { Mayor control de subjetividad y } \\
\text { más ideas. }\end{array}$ & $\begin{array}{l}\text { Esfuerzo organizativo y de coor- } \\
\text { dinación. }\end{array}$ \\
\hline $\begin{array}{l}\text { Evaluación de análisis por } \\
\text { personas externas tras el con- } \\
\text { trol analítico de todo el equipo } \\
\text { de investigación }\end{array}$ & $\begin{array}{l}\text { Mejora del análisis y de su } \\
\text { comunicación, además de velar } \\
\text { por la validez interna respecto } \\
\text { a la metodología y análisis } \\
\text { realizados. Vela por la calidad } \\
\text { y validez general de la investi- } \\
\text { gación. }\end{array}$ & Esfuerzo económico, y relacional. \\
\hline $\begin{array}{l}\text { Información de archivo y tras- } \\
\text { tienda disponible y accesible }\end{array}$ & $\begin{array}{l}\text { Transparencia, responsabilidad } \\
\text { social con la academia y la } \\
\text { ciudadanía en general. (Tríptico } \\
\text { informativo, cuestionario so- } \\
\text { ciodemográfico, guiones de las } \\
\text { entrevistas y códigos utilizados } \\
\text { en la codificación) }\end{array}$ & $\begin{array}{l}\text { Preservar la confidencialidad y } \\
\text { el anonimato de los datos perso- } \\
\text { nales. }\end{array}$ \\
\hline
\end{tabular}

El presente artículo pensamos que genera una forma de promover y mejorar la calidad, en la línea propuesta por Valles (2015). Aquí se utiliza el archivo y la trastienda con un sentido vinculado a la historia de la investigación y a todas las decisiones adoptadas sobre la misma, ofreciendo evidencias así como materiales 
y documentación utilizada, en definitiva siendo transparentes sobre el proceso metodológico llevado a cabo.

A la validez interna se contribuye por la credibilidad obtenida y la confiabilidad del proceso de investigación, como criterios de calidad de la investigación cualitativa para Ruíz de Olabuenaga (2012). Aquí se explicita todo el proceso de investigación realizado como aspecto fundamental, proceso congruente entre el conocimiento que genera, las prácticas y la experiencia de investigación, explicándose también las decisiones previamente tomadas, las planificadas y las adoptadas posteriormente.

La amplitud de la muestra y la cantidad de datos empíricos procedentes de la entrevista conjunta a la pareja en la primera ola, y de las entrevistas por separado en la primera y segunda ola, fue posible por haber trabajado un equipo de diez investigadores que han participado conjuntamente en todas y cada una de las fases de investigación. Esto ha permitido que todo el equipo enriquezca el proceso investigador y vele por la calidad de la investigación llevada a cabo, ya que la continua discusión y puesta en común conjunta de todas las fases del proceso era la práctica y forma de proceder habitual.

Sobre el proceso de contactación y su esmerado cuidado, la información que se les daba a las parejas al inicio de la contactación y la que aparecía en el tríptico informativo difundido era muy general. Se indicaba que estábamos interesados en las parejas que actualmente tienen hijos y cómo un hijo influye en sus vidas cotidianas, sin que en ningún momento se mencionase el concepto género, o que nos interesara la división de género o las relaciones de género y sus cambios. Este aspecto se tuvo especialmente en cuenta durante toda la contactación, precisamente para no condicionar a las parejas desde el inicio, ya que esto hubiera excluido de la autoselección a parejas con un reparto alejado del ideal igualitario, o bien, habría condicionado previamente los posibles discursos de las parejas que aceptasen participar en la investigación. De ahí, que en la muestra, a pesar de ser todas ellas parejas de doble ingreso activas, hubiese casi una mitad de las parejas con ideales de interacción de parejas igualitario y otra mitad con una ideal de pareja más tradicional respecto a los roles desempeñados por varones y mujeres en la vida cotidiana, algo que pudo conocerse tras analizar las entrevistas.

La conciencia sobre la importancia de la fidelización ha permitido cuidar la relación previa y posterior a las entrevista de la primera ola, garantizando la presencia de la misma muestra para la segunda fase, aunque se hayan perdido diez parejas por motivos que se han justificado, externos al equipo de investigación.

El análisis realizado por el equipo ha permitido trabajar con los discursos completos de las entrevistas, habiendo sido el proceso de análisis complejo. Los análisis de discurso realizados por los subequipos han sido evaluados en primer lugar por todo el equipo conjuntamente, y en segundo lugar por personas expertas en investigación cualitativa y en los temas objeto de análisis, externas al

\footnotetext{
${ }^{5}$ Información disponible en la página web "http://transparent.upf.edu/".
} 
equipo de investigación. Esta estrategia ha sido adoptada para velar y mejorar la validez y la calidad de los análisis realizados.

Finalmente, y con el objetivo de analizar de forma crítica las ventajas y limitaciones de la ICL realizada, queremos centrarnos en dos cuestiones críticas aún no tratadas, referidas a la censura estructural y a la saturación de los discursos. En toda situación social se dan procesos de censura estructural, véase Bourdieu (1985) y más aún en las situaciones de entrevista, ya que cada sujeto entrevistado de alguna forma intenta agradar a quien le entrevista y trata de ofrecer la mejor imagen de sí mismo. Por tanto, es el transcurrir del discurso el que va intentado desvelar la credibilidad de los discursos para su posterior interpretación y comprensión. Con Ortí (2014) pensamos que la interpretación adquiere sentido cuando reconstruye e interpreta de acuerdo con los objetivos de investigación. La investigación cualitativa longitudinal ofrece más oportunidades de reflexionar sobre la credibilidad de los discursos y la subjetividad de quien investiga. Entrevistar a los mismos sujetos en un segundo momento permite atenuar en parte los efectos de la censura estructural, al poder confrontar a la persona con sus contradicciones, ambivalencias, imprecisiones, en definitiva con su previsión y su posterior realidad. En las entrevistas conjuntas está más presente la censura estructural, pues hay un posicionamiento ante la pareja y ante quien le entrevista. En las entrevistas por separado, la persona entrevistada se siente con mayor libertad para expresar sus malestares o su punto de vista con respecto al curso que va tomando su vida y su relación de pareja -hay menos censura estructural. Por ejemplo, en un caso se percibió claramente la censura estructural que ejerció uno de los cónyuges -el marido- sobre el otro cónyuge -la mujer- durante la entrevista conjunta (para un mayor desarrollo de los efectos de la censura estructural en las entrevistas conjuntas, véase Botía-Morillas 2013). Observar esta dinámica ha permitido comprender mejor las actitudes subyacentes de esta persona, contrarias a la imagen que de sí mismo quería transmitir. En otras ocasiones, las entrevistas conjuntas han permitido observar la forma de interactuar y negociar en la pareja, lo que informa de las dinámicas implícitas de poder (Komter 1989). También se pueden observar las diferencias de percepciones y las justificaciones que cada miembro de la pareja da a cada tema tratado. Los problemas ligados a la censura estructural se han podido contrarrestar también por la existencia de entrevistas individuales, que han permitido a su vez acceder mejor a la sinceri$d a d$ reflexiva individual, ya que cada sujeto tiene una concepción diferente de la realidad social vivida. El análisis de las entrevistas de ella, de él y de ambos ha permitido validar mejor los discursos desarrollados por cada sujeto entrevistado en sus diferentes entrevistas.

En cuanto al criterio de saturación se puede afirmar que contar con una muestra cualitativa tan amplia, 68 parejas entrevistadas en la primera ola y 58 en la segunda ola ha permitido aproximarnos al proceso de saturación de los discursos, en los temas y dimensiones centrales del análisis realizado, como criterio de valor y calidad de la investigación cualitativa (Callejo 1998; Bertaux 1993). El nivel de saturación puede entenderse como el momento a partir del cual nuevas entrevistas no añaden algo nuevo, sustancialmente relevante respecto de las re- 
laciones socioestructurales que atraviesan a los sujetos entrevistados, como para que se justifique la ampliación de la muestra.

Ciertamente a pesar de la diversidad muestral dentro de su homogenidad -parejas heterosexuales, bi-activas, esperando su primer bebé-, que componen dos submuestras más homogéneas entre sí, una de mayor tamaño de clase media y otra de menor tamaño de clase trabajadora, ambas con pautas laborales parecidas, puede afirmarse la aproximación al nivel de saturación (Bertaux 1993) de los discursos. Esto significa que si bien los resultados obtenidos no pueden ser generalizados al total de parejas españolas, puesto que existe gran diversidad de modelos parentales, y aquí solamente nos hemos centrado en uno de ellos, sí son resultados consistentes para parejas de similar perfil a las entrevistadas. Sin que podamos afirmar la validez externa garantizada, ya que la saturación de los discursos es más un criterio de aproximación, que un criterio absoluto. Para concluir, se puede afirmar que una investigación cualitativa longitudinal como la presentada tiene una gran potencialidad analítica para contextos sociales de parejas de doble ingreso en España.

\section{REFERENCIAS BIBLIOGRÁFICAS}

ABRIL, Paco; AMIGOT, Patricia; BOTÍA-MORILLAS, Carmen; DOMÍNGUEZFOLGUERAS, Marta; GONZÁLEZ, María José; JURADO-GUERRERO, Teresa; LAPUERTA, Irene; MARTÍN-GARCÍA, Teresa; MONFERRER, Jordi; y SEIZ, Marta (2015). "Ideales igualitarios y planes tradicionales: análisis de parejas primerizas en España”, REIS, Revista Española de Investigaciones Sociológicas, 150, pp. 3-22.

ALBERDI, Inés (2003): "El trabajo remunerado de las mujeres y su impacto en la vida familiar", Arbor, 694, pp. 195-238.

ALONSO Benito, Luis Enrique (1998): “Análisis sociológico de los discursos: una aproximación desde los usos concretos", en La mirada cualitativa en sociología Madrid, Fundamentos, pp. 187-220.

ALONSO Benito, Luis Enrique y RODRÍGUEZ, Victoriano (2014): “La génesis sociohistórica del cualitativismo crítico español. Una perspectiva de investigación comprometida con la emancipación social", Arxius, 31, pp. 13-26.

BERTAUX, Daniel (1993): “La perspectiva biográfica: validez metodológica y potencialidades", en La historia oral: métodos y experiencias, Madrid, Debate, pp. 149-161.

BERTAUX, Daniel y BERTAUX-WIAME Isabelle (1993): "Historias de vida del oficio de panadero", en La historia oral: métodos y experiencias, Madrid, Debate, pp. 231250.

BOTÍA-MORILLAS, Carmen (2011): “Maternidades y paternidades en transición. Dinámica y tensiones en las relaciones de género en parejas heterosexuales no tradicionales en los cuidados de su primer hijo". (Tesis doctoral inédita, dirigida por Enrique Martín Criado). Universidad de Sevilla, Departamento de Sociología.

BOTÍA-MORILLAS, Carmen (2013): “Cómo diseñar una investigación para el análisis de las relaciones de género. Aportaciones metodológicas”. Papers, Revista de Sociología, 98 (3), pp. 443-470.

BOURDIEU, Pierre (1985): ¿Qué significa hablar?: Economía de los intercambios lingüísticos, Madrid, Akal. 
CAÏS, Jordi; FOLGUERA, Laia; FORMOSO, Climent (2014): Investigación cualitativa longitudinal, Madrid, Centro de Investigaciones Sociológicas.

CALLEJO, Javier (1998): "Los límites de la formalización de las prácticas cualitativas de investigación social: La saturación", Sociológica. Revista de pensamiento social, 3, pp. 93-120.

COLECTIVO IOÉ (2011). Inserción en la escuela española del alumnado inmigrante iberoamericano. Análisis longitudinal de trayectorias de éxito y fracaso, Madrid, OIE.

CONDE, Fernando (2008): "Los grupos triangulares como "espacios transicionales" para la producción discursiva: Un estudio sobre la vivienda en Huelva", en Estrategias y prácticas cualitativas de investigación social, Madrid: Pearson Educación, pp. 155188.

DEMA, Sandra (2005): "Entre la tradición y la modernidad. Las parejas españolas de doble ingreso", Papers, Revista de Sociología, 77, pp. 135-155. doi.org/10.5565/rev/ papers/v77n 0.934

DEMA, Sandra (2006): Una pareja dos salarios. El dinero y las relaciones de poder en las parejas de doble ingreso, Madrid, Centro de Investigaciones Sociológicas.

DÍAZ, Capitolina; DÍAZ, Cecilia; DEMA, Sandra e IBÁŇEZ, Marta (2004): Dinero, amor e individualización: las relaciones, económicas en las parejas/familias contemporáneas, Oviedo, Instituto Asturiano de la Mujer y KRK ediciones. doi: $10.2307 / 40184782$

FOX, Bonnie (2009): When couples become parents: the creation of gender in the transition to parenthood, Toronto, Canada, University of Toronto Press.

GARRIDO, Luis (2004): Demografía longitudinal de la ocupación, Información Comercial Española, 815, pp. 105-142.

GOFFMAN, Erving (1963): Behavior in Public Places, Nueva York, The Free Press of Glencoe.

GOFFMAN, Erving (1974): Frame Analysis. An Essay on the Organization of Experience, Nueva York, Harper \& Row. (reed. 1986, Boston, Northeastern University Press).

GONZÁLEZ, Ma José, y JURADO-GUERRERO, Teresa (ed.) (2015) Padres y Madres corresponsables, una utopía real, Madrid, La Catarata

GUTIÉRREZ, Marta (2002): “Triangular público, doméstico y privado, o ¿cómo negociar en pareja?, Revista Española de Investigaciones Sociológicas, 99, pp. 61-86.

HAMMERSLEY, Martyn y ATKINSON, Paul (1994): Etnografía. Métodos de investigación, Paidós, Barcelona.

HOCHSCHILD, Arlie y MACHUNG Anne (1989): The second shift: Working parents and the revolution at home, New York, Penguin.

IBÁÑNEZ, Jesús (1979): Más allá de la sociología: el grupo de discusión, teoría y crítica, Madrid, Siglo XXI.

JURADO-GUERRERO, Teresa; Mª José GONZÁLEZ; y Manuela NALDINI, (2012). Gender, Family and the Labour Market in Post-industrial Societies: A New Social Compromise?, en BURRONI, L.; KEUNE, M. y MEARDI G. (Eds): Economy and Society in Europe: A Relationship in Crisis?, Cheltenham, Edward Elgarm, pp.6180.

KOMTER, Aafke (1989): "Hidden power in marriage", Gender \& Society, 3, pp. 187216.

LEAHY, Mary y DOUGHNEY, James (2006): "Women, work and preference formation: A critique of Catherine Hakim's Preference Theory", Journal of Business System, Governance and Ethics, 1 (1), pp. 37-48. 
doi. $10.15209 /$ jbsge.v1i1.79

LUCAS, Ángel de (1992): Actitudes y representaciones sociales de la población de la Comunidad de Madrid en relación con los Censos de Población y Vivienda de 199, Madrid, Consejería de Economía.

MARTÍN CRIADO, Enrique (1997): "El grupo de discusión como situación social”, Revista Española de Investigaciones Sociológicas, 79, pp. 81-112.

MARTÍN CRIADO, Enrique (1998): "Los decires y los haceres", Papers, Revista de Sociología, 56, pp. 57-71.

MARTÍN CRIADO, Enrique (2004): "El valor de la buena madre. Oficio de ama de casa, alimentación y salud entre las mujeres de clases populares", Revista Española de Sociología, 4 (2), pp. 93-118.

MARTÍN CRIADO, Enrique (2014a): "Describir, explicar, participar en el debate público. La necesidad de la investigación cualitativa", Arxius, 31, pp. 85-96.

MARTÍN CRIADO, Enrique (2014b): "Mentiras, inconsistencias y ambivalencias. Teoría de la acción y análisis de discurso", Revista Internacional de Sociología, 72 (1), pp. 115-138. doi: 10.3989/ris.2012.07.24

MERLLIÉ, Dominique (1993): La construcción estadística, en Iniciación a la práctica sociológica, México, Siglo XXI.

MERTON, Robert K. (1972): Teoría y estructura sociales, México, Fondo de Cultura Económica.

ORTÍ, Alfonso (2001): "En el margen del centro: la formación de la perspectiva sociológica crítica de la generación de 1956”, Revista Española de Sociología, 1, pp. 119166.

ORTÍ, Alfonso (2007): "Veinticinco años después: el oficio de sociólogo en la España plural", Revista Española de Sociología, 7, pp. 27-75.

ORTÍ, Alfonso (2014): "Encuestación cualitativa y praxis socioinstitucional. De la configuración de "subjetividades sociales" a la de "discursos virtuales", Arxius, pp. $27-$ 56.

PRIETO, Carlos (2015): Trabajo, cuidados, tiempo libre y relaciones de género en la sociedad española, Madrid, Ediciones Cinca.

RUIZ DE OLABUÉNAGA, José Ignacio (2012): Metodología de la investigación cualitativa, Bilbao, Universidad de Deusto.

TAYLOR, Steve y BOGDAN, Robert (2002): Introducción a los métodos cualitativos de investigación, Madrid, Paidós.

TIMMERMANS, Stefan y TAVORY Iddo, (2012): “Theory construction in qualitative research from grounded theory to abductive analysis", Sociological Theory, 30, pp. 167-186.

TOBÍO, Constanza (2001): "Conciliación o contradicción: cómo hacen las madres trabajadoras”, Revista Española de Investigaciones Sociológicas, 97, pp. 155-188.

TOBÍO, Constanza (2005): Madres que trabajan. Dilemas y estrategias, Madrid, Cátedra.

VALLES, Miguel S. (2015): "Prácticas pioneras de archivo en la investigación sociológica cualitativa en España”, Revista Española de Investigaciones Sociológicas, 150, pp. 173-190. doi:10.5477/cis/reis

WIESMANN, Stephanie, BOEIJE, Hennie; DOORNE-HUISKES, Anneke van y DULK, Laura den (2008): "Not worth mentioning': The implicit and explicit nature of decision-making about the division of paid and domestic work", Community, Work \& Family, 11, pp. 341-363. doi:10.1080/13668800802361781

ZÁRRAGA, José Luis (2009): "Por qué, qué y para qué investigamos: la trastienda de la investigación social”, Política y Sociedad, 46, 3, pp. 37-56. 

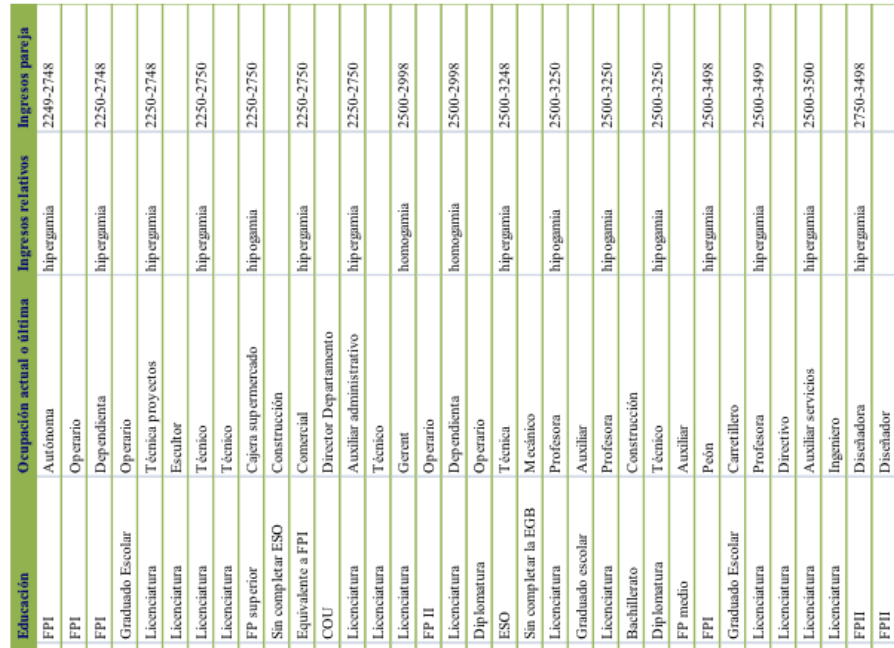



踀

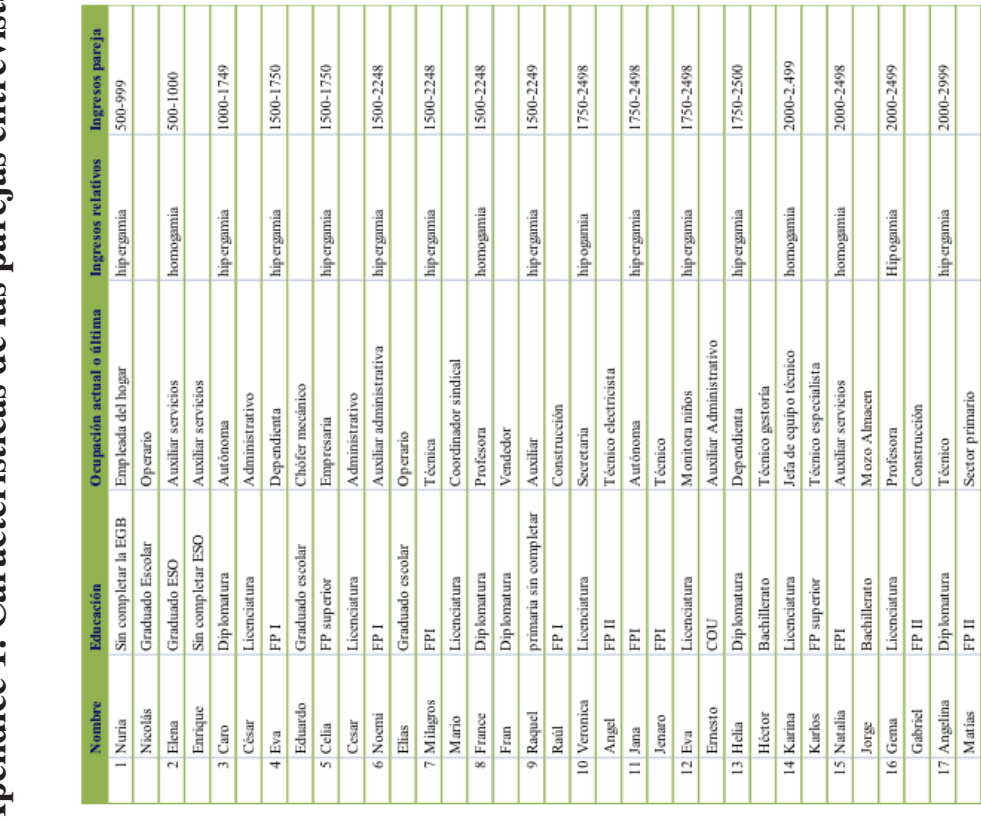

EMPIRIA. Revista de Metodología de Ciencias Sociales. N. ${ }^{\circ} 41$ septiembre-diciembre, 2018, pp. 33-56, ISSN: 1139-5737, DOI/empiria.41.2018.22603 



EMPIRIA. Revista de Metodología de Ciencias Sociales. N. ${ }^{4} 41$ septiembre-diciembre, 2018, pp. 33-56. ISSN: 1139-5737, DOI/empiria.41.2018.22603 